\title{
Increased Soluble Cytoplasmic Bcl-2 Protein Serum Levels and Expression and Decreased Fas Expression in Lymphocytes and Monocytes in Juvenile Dermatomyositis
}

\author{
Bernadete L. Liphaus, Adriana E.M. Sallum, Nadia E. Aikawa, Maria Helena B. Kiss, \\ Solange Carrasco, Patricia Palmeira, Laila Lima, Clovis A. Silva, Claudia Goldenstein-Schainberg, \\ and Magda Carneiro-Sampaio
}

\begin{abstract}
Objective. To evaluate soluble Fas antigen (sFas), sFas ligand (sFasL), soluble tumor necrosis factor-related apoptosis-inducing ligand, and soluble cytoplasmic $\mathrm{Bcl}-2$ protein (sBcl-2) serum levels, Fas and Bcl-2 expressions in T and B lymphocytes and monocytes and relations with erythrocyte sedimentation rate, C-reactive protein (CRP), Childhood Myositis Assessment Scale, and manual muscle testing in juvenile dermatomyositis (JDM).

Methods. Serum levels were determined by ELISA and peripheral cell expressions by flow cytometry for patients with JDM or juvenile idiopathic arthritis (JIA), and healthy controls.

Results. Patients with JDM had increased sBcl-2, which correlated with CRP. Expression of Bcl-2 was increased and expression of Fas was decreased in CD3+, CD4+, and CD8+ T lymphocytes compared with JIA and/or healthy controls.

Conclusion. Patients with JDM presented a unique apoptosis-related proteins profile, which may contribute to disease development. (First Release August 1 2018; J Rheumatol 2018;45:1577-80; doi:10.3899/jrheum.171248)
\end{abstract}

Key Indexing Terms: APOPTOSIS

JUVENILE DERMATOMYOSITIS LYMPHOCYTES
SOLUBLE BCL-2
MONOCYTES
From the Laboratory of Medical Investigation, and Pediatric Rheumatology Unit, Children's Institute, and Hospital das Clínicas HCFMUSP, Faculdade de Medicina, Universidade de São Paulo, São Paulo, Brazil.

Financial support from the São Paulo Research Foundation (FAPESP grants 2008/58238-4 and 2012/22997-4).

B.L. Liphaus, MD, PhD, Laboratory of Medical Investigation, Children's Institute, Faculdade de Medicina, Universidade de São Paulo;

A.E. Sallum, MD, PhD, Pediatric Rheumatology Unit, Children's Institute, Faculdade de Medicina, Universidade de São Paulo; N.E. Aikawa, MD, PhD, Pediatric Rheumatology Unit, Children's Institute, Faculdade de Medicina, Universidade de São Paulo; M.H. Kiss, MD, PhD, Faculdade de Medicina, Universidade de São Paulo; S. Carrasco, MS, Hospital das Clínicas HCFMUSP, Faculdade de Medicina, Universidade de São Paulo; P. Palmeira, PhD, Hospital das Clínicas HCFMUSP, Faculdade de Medicina, Universidade de São Paulo; L. Lima, BsC, Laboratory of Medical Investigation, Children's Institute, Faculdade de Medicina, Universidade de São Paulo; C.A. Silva, MD, PhD, Pediatric Rheumatology Unit, Children's Institute, and Hospital das Clínicas HCFMUSP, Faculdade de Medicina, Universidade de São Paulo; C. Goldenstein-Schainberg, MD, PhD, Hospital das Clínicas HCFMUSP, Faculdade de Medicina, Universidade de São Paulo; M. Carneiro-Sampaio, MD, PhD, Professor, Laboratory of Medical Investigation, Children's Institute, Faculdade de Medicina, Universidade de São Paulo.

Address correspondence to Dr. B.L. Liphaus, Instituto da Criança, Faculdade de Medicina, Universidade de São Paulo, Av. Dr. Eneas de Carvalho Aguiar 647, $5^{\circ}$ andar, LIM 36, Cerqueira Cesar, São Paulo-SP, Brazil, CEP 05403-900.E-mail: bernadete.liphaus@hc.fm.usp.br Accepted for publication May 25, 2018.
Juvenile dermatomyositis (JDM) pathogenesis involves cell-mediated and humoral immune responses and is distinct from that of polymyositis and dermatomyositis ${ }^{1,2,3}$.

Fas, Fas ligand (FasL), and tumor necrosis factor-related apoptosis-inducing ligand (TRAIL) are apoptosis-promoting proteins. In turn, through a different pathway, cytoplasmic protein Bcl-2 protects cells from death. These protein expressions were demonstrated in muscle fibers and infiltrating inflammatory cells from patients with $\mathrm{JDM}^{4,5,6,7,8}$. Our group has observed altered soluble (s)Fas, sTRAIL, and sFasL levels, as well as expressions of Fas and Bcl-2 in lymphocytes and monocytes from patients with juvenile systemic lupus erythematosus (JSLE) $9,10,11,12$.

Because altered apoptosis-related protein serum levels ${ }^{13,14}$ and muscle fiber expressions $s^{4,5,6,7,8}$ have been observed, our study simultaneously evaluated sFas, sFasL, sTRAIL, and sBcl-2 serum levels; Fas and Bcl-2 expressions in peripheral $\mathrm{T}$ and $\mathrm{B}$ lymphocytes and monocytes, and their relationships with disease activity variables in patients with JDM.

\section{MATERIALS AND METHODS}

Our cross-sectional study randomly enrolled 34 patients fulfilling Bohan and Peter criteria for definite JDM ${ }^{15}, 40$ sex- and age-matched unrelated healthy individuals, and 33 juvenile idiopathic arthritis (JIA) disease controls

Personal non-commercial use only. The Journal of Rheumatology Copyright $\subset$ 2018 . All rights reserved. 
(15 systemic, 11 polyarticular, and 7 oligoarticular) ${ }^{16}$. Informed consent was obtained from parents and participants after approval by the CAPPesq ethical committee (No. 195.419). Subjects under suspicion of infection were excluded. Medical records were retrospectively evaluated regarding demographic, clinical, laboratory, and treatment data. Disease activity variables included erythrocyte sedimentation rate (ESR, by Westergren), C-reactive protein (CRP, by nephelometry), Childhood Myositis Assessment Scale (CMAS, range 0-52), and manual muscle testing (MMT, range $0-80)^{17,18}$.

Soluble apoptosis-related protein serum levels were quantified using ELISA kits and following manufacturers' instructions as detailed in our previous work ${ }^{9}$. Duplicate undiluted or $100 \times$ diluted samples and standards were run in parallel for patients and controls. Optical density was measured in spectrophotometer at $450 \mathrm{~nm}$ and serum levels calculated using each standard curve and dilution factor.

Peripheral mononuclear cells were isolated and stained with monoclonal antibodies, using instruments and procedures detailed in previous studies ${ }^{10,11,12}$. For flow cytometric analysis, lymphocytes were separated by gating of CD3 versus site scatter (SSC) and monocytes by gating CD14 versus SSC. Nonviable cells were excluded from analysis by drawing gates not including events with low forward scatter (FSC) and high SSC light properties. Over study duration, instrument settings and procedures were identical. In each flow cytometry session, both patient and control samples were analyzed. Double-positive cells for Fas or Bcl-2 and CD3, CD4, CD8, CD19, or CD14 were identified by dot-plot histograms and the results are shown as percentage of double-positive cells in relation to the total number of lymphocytes or monocytes in the respective gate and as mean fluorescence intensity (MFI).

The 3 study groups were compared using the Kruskal-Wallis ANOVA test followed by the Mann-Whitney U test for comparisons of the 2 groups. After Bonferroni's correction for multiple comparisons in posthoc analyses, only p values $\leq 0.01$ were considered significant. Results are presented as median and interquartile range (IQR). Spearman's rank test was used for correlations with disease activity variables.

\section{RESULTS}

Patients with JDM (22 girls) had median ages of 9.9 years (range 3.4-20.8), disease duration of 3.4 years (0.2-15.9), CMAS of 48 (28-52), MMT of 80 (33-80), ESR of 16.0 $\mathrm{mm} / \mathrm{h}$ (2.0-55.0), and CRP of $0.7 \mathrm{mg} / \mathrm{l}(0.2-5.7)$. Twenty-two patients were taking oral prednisone, 15 methotrexate, 12 hydroxychloroquine, 4 cyclosporine, 1 cyclophosphamide, and 1 intravenous immunoglobulin. JIA disease controls (20 girls) had median age of 12.5 years (range 3.8-19.2), disease duration of 4.9 years (0.2-14.8), and ESR of $23.0 \mathrm{~mm} / \mathrm{h}$ (2.0-56.0), and were taking antiinflammatory drugs, oral prednisone, and/or immunosuppressive therapy, according to clinical condition. Healthy controls (26 girls) had a median age of 10.9 years (range 2.1-20.3).

Soluble Bcl-2 levels were different among the 3 groups $(\mathrm{p}=0.0004)$ and significantly increased in patients with JDM compared with healthy [median $29.3 \mathrm{ng} / \mathrm{ml}$ (IQR 15.1-39.9) vs $7.4 \mathrm{ng} / \mathrm{ml}$ (IQR 4.4-11.0), $\mathrm{p}=0.0001]$ and JIA [6.4 ng/ml (IQR 3.2-30.5), $\mathrm{p}=0.003$, Figure 1A] controls. Moreover, sBcl-2 levels negatively correlated with CRP $(r=-0.55$; $\mathrm{p}=0.01)$. sFas and sTRAIL levels were similar among groups $(p=0.1$ and $p=0.9$, respectively, Figure 2$)$. Soluble FasL was detected in 1 patient with JDM $(0.32 \mathrm{ng} / \mathrm{ml})$, contrasting with $60 \%$ in healthy and $33 \%$ in JIA controls
(Figure 2). No correlation was observed with CMAS, MMT, or ESR.

In addition, proportion of $\mathrm{Bcl}-2-$ expressing $\mathrm{CD} 3+\mathrm{T}$ lymphocytes was different among the 3 groups $(p=0.01)$ and significantly higher in patients with JDM compared with healthy controls [median $60.7 \%$ (IQR 56.3-71.3) vs $52.1 \%$ (IQR 46.7-55.3), $\mathrm{p}=0.007$, Figure 1B]. As well, proportion of Bcl-2-expressing CD4+ T cells was different among groups $(\mathrm{p}=0.003)$ and increased compared with healthy controls [median 36.3\% (IQR 33.9-45.8) vs 27.8\% (IQR 23.9-32.2), $\mathrm{p}=0.0007]$ but not JIA controls [32.0\% (IQR 26.3-36.5), $\mathrm{p}=0.03$, Figure $1 \mathrm{C}]$ considering Bonferroni's correction. Moreover, expression levels of Bcl-2 in CD8+ T lymphocytes were different among groups $(p=0.007)$ and increased in patients with JDM compared with healthy controls [median MFI 32.4 (IQR 24.5-37.3) vs 23.1 (IQR 21.1-25.9), $\mathrm{p}=0.004$ ] and JIA controls [median MFI 24.4 (IQR 20.6-27.9), p = 0.006, Figure 1D]. Expressions of Bcl-2 in CD19+ B lymphocytes and CD14+ monocytes were similar in patients with JDM, healthy controls, and JIA controls. Surprisingly, expressions of Bcl-2 in T and B lymphocytes and monocytes did not correlate with ESR, CRP, CMAS, or MMT.

Otherwise, the proportion of Fas-expressing CD3+ T lymphocytes was different among the 3 groups $(\mathrm{p}=0.03)$ and significantly decreased in patients with JDM compared with JIA controls [median 23.8\% (IQR 20.6-56.5) vs 32.8\% (IQR 28.3-36.4), $\mathrm{p}=0.01]$. Proportion of Fas-expressing CD4+ T lymphocytes was also decreased [median 14.1\% (IQR 12.8-16.8) vs $17.2 \%$ (IQR 14.5-20.0), $\mathrm{p}=0.01$ ] compared with JIA controls. No difference was observed in proportion of Fas-expressing CD8+ T lymphocytes after Bonferroni's correction [median JDM 7.6\% (IQR 5.5-14.6) vs JIA $13.6 \%$ (IQR 11.9-20.4), p = 0.03; Figure 2]. Expressions of Fas in B lymphocytes and monocytes were similar to those of healthy and JIA controls. No correlation was observed between Fas-expressing cells and disease activity variables.

Patients with JIA had soluble apoptosis-protein levels similar to those of healthy controls (Figure 1A and Figure 2), and considering Bonferroni's correction, differences in proportion of Bcl-2-expressing and Fas-expressing cells were not significant (Figure 1B-E and Figure 2).

\section{DISCUSSION}

Studies regarding apoptosis-related proteins in patients with JDM are few; data are not conclusive and whether serum levels depict their expression in which cell types is not fully understood $^{7,11,13,14,19}$. Our study results parallel previous reports of Bcl-2 overexpression in muscle fibers ${ }^{4,6,7}$. Thus we suggest that increased $\mathrm{sBcl}-2$ levels reflect higher expression of Bcl-2 not only in myofibers but also in peripheral cells, because increased expression of Bcl-2 was shown in all peripheral T lymphocyte subsets. Previous findings and correlation with CRP support a hypothesis of an apoptosis role in

Personal non-commercial use only. The Journal of Rheumatology Copyright $\subset$ $\subset$ 2018. All rights reserved 

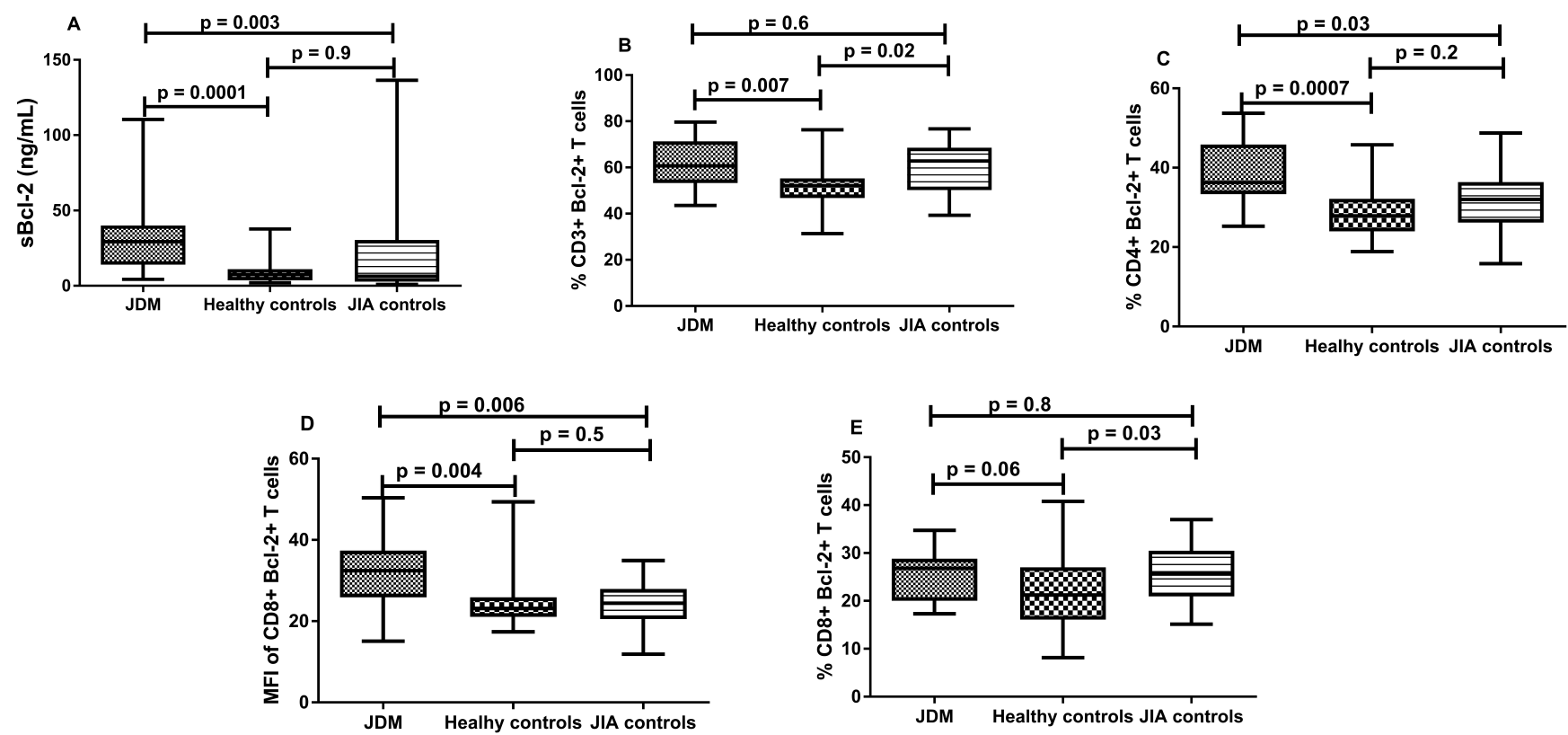

Figure 1. Soluble Bcl-2 levels and expressions of Bcl-2 in lymphocytes among the 3 groups in the study. JDM: juvenile dermatomyositis; JIA: juvenile idiopathic arthritis; MFI: mean fluorescence intensity.
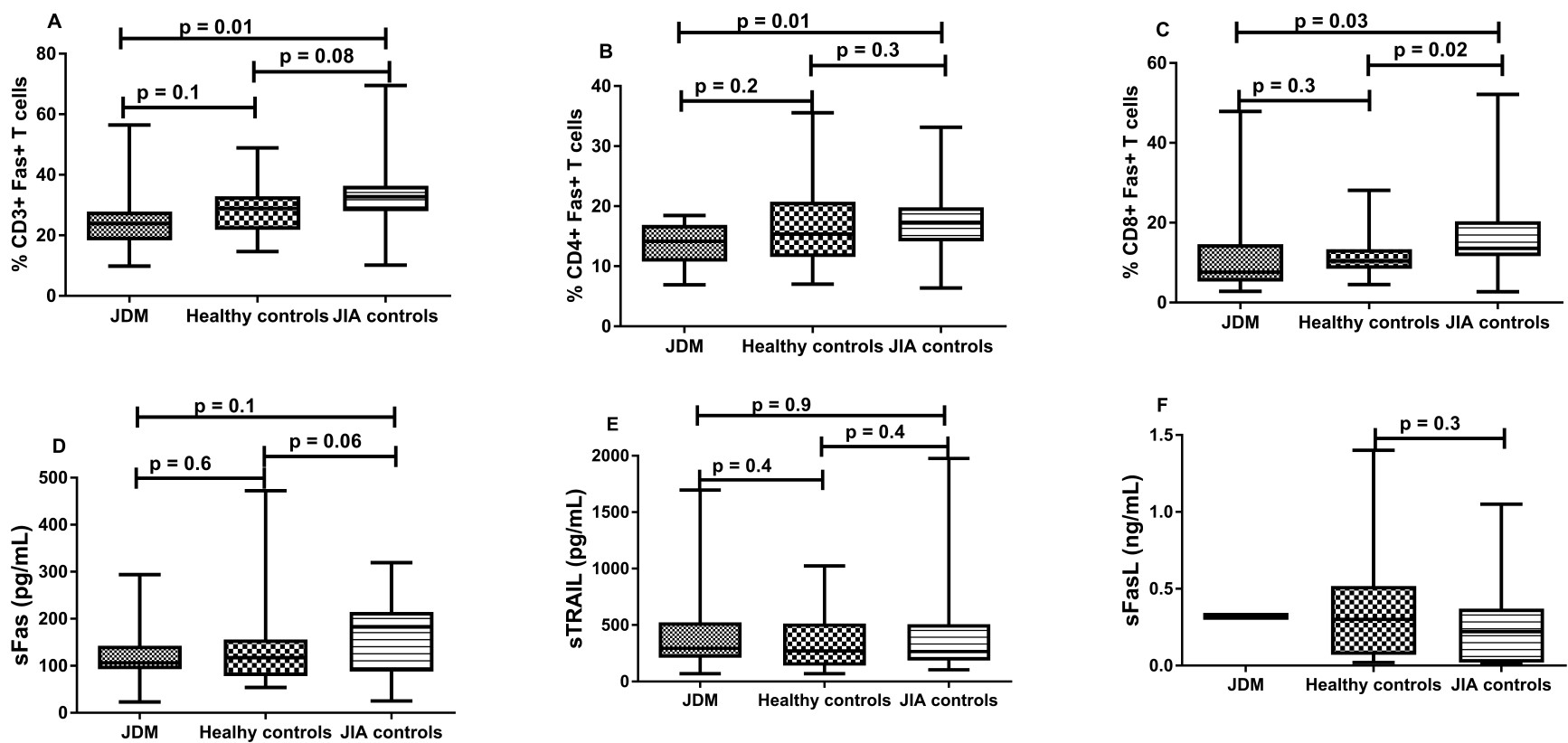

Figure 2. Proportion of CD3+, CD4+, and CD8+ Fas+ T cells, and sFAS, sTRAIL, and sFasL levels in the 3 groups in the study. sFAS: soluble Fas antigen; sTRAIL: soluble tumor necrosis factor-related apoptosis-inducing ligand; sFasL: sFAS ligand; JDM: juvenile dermatomyositis; JIA: juvenile idiopathic arthritis.

disease development, which may lead to persistence of self-reactive lymphocyte clones and thus to autoimmunity, if Bcl-2 expressing cells remain viable ${ }^{20}$. Otherwise, Bcl-2 overexpression can protect myofibers from death and may be part of a regeneration program ${ }^{8}$. Therefore, further studies are needed to elucidate Bcl-2 protein function in JDM.
Increased Fas expression was observed in muscle fibers and infiltrating CD4+ and CD8+ T cells in polymyositis and dermatomyositis ${ }^{4,6,8}$. In addition, Fas, TRAIL, and caspase-3 were identified in muscle biopsies in $\mathrm{JDM}^{5}$. Decreased proportion of Fas-expressing peripheral CD4+ and CD8+ T lymphocytes reinforces the idea of a pathogenic role not 
only for Bcl-2 but also for Fas protein in JDM.

Interestingly, patients with JDM had expressions of Fas and Bcl-2 in B lymphocytes and monocytes similar to controls, contrasting with JSLE patients, who presented decreased expressions of Fas and $\mathrm{Bcl}-2^{12}$ and impaired apoptotic debris removal by monocytes ${ }^{20}$, and increased expression of Fas in B lymphocytes ${ }^{10,11}$.

Because steroids and immunosuppressive drugs are known to interfere with cell apoptosis and to both increase or decrease apoptosis-related protein expressions and serum levels ${ }^{5,13,14,19}$, that sort of influence cannot be ruled out in our results.

Soluble Fas and sTRAIL levels were similar to healthy and JIA controls and did not correlate with ESR, CRP, CMAS, or MMT, though other authors have observed increased sFas levels in patients with JDM ${ }^{13,14}$. Consistent with a previous study ${ }^{14}$, sFasL was undetectable in a great number of patients with JDM. Hence, the sFas, sTRAIL, and sFasL roles in JDM pathogenesis and their use as biomarkers (as in patients with JSLE) ${ }^{9}$ still needs clarification.

Although studies of patients with rheumatoid arthritis and JIA showed increased sFas and sFasL levels ${ }^{13,14}$, this inflammatory control group had cellular expressions and soluble levels of apoptosis-related proteins similar to healthy controls, reinforcing that the alterations observed herein are somewhat specific for patients with JDM.

Our study's limitation was that FSC/SSC gating excluded debris but not all nonviable cells, and these may have contributed to the apoptotic signatures described. Otherwise the study differentials were to solely evaluate patients with definite JDM, to analyze both apoptosis-inducing and inhibiting proteins not only in $\mathrm{T}$ and $\mathrm{B}$ lymphocytes but also in monocytes, and to simultaneously evaluate soluble forms, which gave the opportunity to draw a profile of apoptosis-related proteins in JDM. However, whether these results can be considered a primary pathogenic event or alternatively a secondary epiphenomenon remains to be elucidated.

Therefore, patients with JDM present an imbalance of apoptosis-related proteins, as previously shown for other autoimmune diseases, not only in muscle fibers but also in Bcl-2 and Fas peripheral lymphocyte expressions as well as serum levels, which may contribute to disease development.

\section{ACKNOWLEDGMENT}

The authors are grateful to all patients for their kind participation.

\section{REFERENCES}

1. Sallum AM, Kiss MH, Sachetti S, Resende MB, Moutinho KC, Carvalho Mde S, et al. Juvenile dermatomyositis: clinical, laboratorial, histological, therapeutical and evolutive parameters of 35 patients. Arq Neuropsiquiatr 2002;60:889-99.

2. Sallum AM, Kiss MH, Silva CA, Wakamatsu A, Sachetti S, Lotufo $\mathrm{S}$, et al. MHC class I and II expression in juvenile dermatomyositis skeletal muscle. Clin Exp Rheumatol 2009;27:519-26.

3. Sallum AM, Kiss MH, Silva CA, Wakamatsu A, Vianna MA, Sachetti S, et al. Difference in adhesion molecule expression (ICAM-1 and VCAM-1) in juvenile and adult dermatomyositis, polymyositis and inclusion body myositis. Autoimmun Rev 2006;5:93-100

4. Behrens L, Bender A, Johnson MA, Hohlfeld R. Cytotoxic mechanisms in inflammatory myopathies - Co-expression of Fas and protective Bcl-2 in muscle fibres and inflammatory cells. Brain 1997;120:929-38.

5. Zhao Y, Fedczyna TO, McVicker V, Caliendo J, Li H, Pachman LM Apoptosis in the skeletal muscle of untreated children with juvenile dermatomyositis: Impact of duration of untreated disease. Clin Immunol 2007;125:165-72.

6. Sugiura T, Murakawa Y, Nagai A, Kondo M, Kobayashi S. Fas and Fas ligand interaction induces apoptosis in inflammatory myopathies - CD4(+) T cells cause muscle cell injury directly in polymyositis. Arthritis Rheum 1999;42:291-8.

7. Falcini F, Calzolari A, Generini S, Pignone A, Simonini G, Zulian F, et al. Bcl-2, p53 and c-myc expression in juvenile dermatomyositis. Clin Exp Rheumatol 2000;18:643-6.

8. De Bleecker JL, Meire VI, Van Walleghem IE, Groessens IM, Schroder JM. Immunolocalization of FAS and FAS ligand in inflammatory myopathies. Acta Neuropathol 2001;101:572-8.

9. Liphaus BL, Kiss MHB, Carrasco S, Palmeira P, Goldenstein-Schainberg C, Carneiro-Sampaio M. Increased serum sFas, sTRAIL and reduced sFasL in juvenile-onset systemic lupus erythematosus. Clin Rheumatol 2017;36:2847-52.

10. Liphaus BL, Kiss MH, Carrasco S, Goldenstein-Schainberg C. Increased Fas and Bcl-2 expression on peripheral mononuclear cells from patients with active juvenile-onset systemic lupus erythematosus. J Rheumatol 2007;34:1580-4

11. Liphaus BL, Kiss MH, Carrasco S, Goldenstein-Schainberg C. Increased Fas and Bcl-2 expression on peripheral blood $\mathrm{T}$ and $\mathrm{B}$ lymphocytes from juvenile-onset systemic lupus erythematosus, but not from juvenile rheumatoid arthritis and juvenile dermatomyositis. Clin Dev Immunol 2006;13:283-7.

12. Liphaus BL, Kiss MH, Carrasco S, Goldenstein-Schainberg C. Reduced expressions of Fas and Bcl-2 proteins in CD14+ monocytes and normal CD14 soluble levels in juvenile systemic lupus erythematosus. Lupus 2013;22:940-7.

13. Sahin M, Aydintug O, Tunc SE, Tutkak H, Naziroglu M. Serum soluble Fas levels in patients with autoimmune rheumatic diseases. Clin Biochem 2007;40:6-10.

14. Nozawa K, Kayagaki N, Tokano Y, Yagita H, Okumura K, Hasimoto H. Soluble Fas (APO-1, CD95) and soluble Fas ligand in rheumatic diseases. Arthritis Rheum 1997;40:1126-9.

15. Bohan A, Peter JB. Polymyositis and dermatomyositis (first of two parts). N Engl J Med 1975;292:344-7.

16. Petty RE, Southwood TR, Manners P, Baum J, Glass DN, Goldenberg J, et al. International League of Associations for Rheumatology classification of juvenile idiopathic arthritis: second revision, Edmonton, 2001. J Rheumatol 2004;31:390-2.

17. Huber AM, Feldman BM, Rennebohm RM, Hicks JE, Lindsley CB, Perez MD, et al. Validation and clinical significance of the Childhood Myositis Assessment Scale for assessment of muscle function in the juvenile idiopathic inflammatory myopathies. Arthritis Rheum 2004;50:1595-603.

18. Rider LG, Koziol D, Giannini EH, Jain MS, Smith MR, Whitney-Mahoney K, et al. Validation of manual muscle testing and a subset of eight muscles for adult and juvenile idiopathic inflammatory myopathies. Arthritis Care Res 2010;62:465-72.

19. Falcini F, Azzari C, Gelli VA, Luchetti M, Gabrielli A, Calzolari A, et al. Reduction of bcl-2 in T cells during immunosuppressive therapy in patients with severe juvenile onset systemic lupus erythematosus. Clin Immunol 1999;93:59-64.

20. Liphaus BL, Kiss MH. The role of apoptosis proteins and complement components in the etiophatogenesis of systemic lupus erythematosus. Clinics 2010;65:327-33. 